\title{
Modélisation du déferlement et de son impact sur les structures côtières
}

\author{
Marcer R. \\ Ingénieur Consultant à PRINCIPIA R.D. \\ Guignard S. \\ Doctorant, PRINCIPIA R.D. et LSEET, Upresa CNRS N ${ }^{\circ} 6017$ \\ Rey V. \\ Maître de Conférence, Upresa CNRS N6017, Université de Toulon et du Var

\section{Résumé}

Des simulations numériques de déferlement de vagues sur des plages et des structures côtières sont réalisées avec un modèle Navier-Stokes diphasique développé par PRINCIPIA (code EOLE). Les équations de Navier-Stokes sont résolues dans les deux phases eau et air et le suivi de la surface libre est assurée par une méthode VOF améliorée (SL-VOF), particulièrement performante pour représenter de fortes déformations des interfaces. Les résultats comparés avec ceux obtenus à partir d'autres modèles simplifiés, basés sur la théorie potentielle, et avec des données expérimentales, montrent les grandes capacités de cette nouvelle approche de modélisation.

\begin{abstract}
Numerical simulations of wave breaking over sloping beaches and on coastal structures were performed by using a Navier-Stokes two phase flow model developed by PRINCIPIA (soft EOLE). The Navier-Stokes equations are solved in air and water, and the interface tracking is achieved by an improved VOF method (SL-VOF method). This method is very accurate when dealing with highly nonlinear interfaces. The results, successfully compared with those obtained by numerical models based on the Boundary Integral Element Method, and with experimental data, show great performances of this new modelling approach.
\end{abstract}

\section{Introduction}

L'étude du comportement d'une vague déferlante sur une plage ou sur une structure fixe ou flottante est d'une importance considérable en océanographie, en ingénierie côtière et offshore, tant au niveau de la connaissance de son influence sur la circulation et le transport sédimentaire dans la bande littorale, que pour l'estimation de ses impacts hydrodynamiques sur les structures.

La simulation numérique du déferlement constitue une difficulté majeure du fait de l'incapacité de la majorité des modèles à simuler de manière réaliste certains phénomènes mis en jeu tels que, par exemple, le suivi des grandes déformations de la 
surface libre de la vague (pouvant aller jusqu'au fractionnement de la crête) ou le couplage des écoulements eau-air, aux densités très différentes.

Différentes approches de modélisation ont été proposées. Les modèles les plus courants sont ceux basés sur la théorie potentielle (voir par exemple [1]). Ils sont généralement très efficaces pour étudier la propagation d'une vague jusqu'au déferlement (zone de shoaling) puisqu'ils allient une grande précision de calcul avec des temps de simulation raisonnables. Par contre, ils sont mis en défaut pour simuler la phase même du déferlement de la vague du fait de leurs hypothèses assez restrictives qui ne permettent pas de représenter les effets rotationnels de l'écoulement, les phénomènes visqueux, ou les aspects multi-interfaces dus aux possibles éclatements de la crête de la vague.

Une approche de modélisation plus précise (mais plus lourde) de type Navier-Stokes avec suivi de la surface libre non-linéaire est donc nécessaire si l'on veut étudier le déferlement d'une vague et ses impacts. Des travaux ont été récemment menés à PRINCIPIA, sur le code de mécanique des fluides EOLE, pour développer un modèle basé sur cette dernière approche. Après une brève description théorique du modèle, plus particulièrement de la méthode de suivi d'interface, on en présente quelques exemples d'applications et de validation.

\section{Présentation du modèle}

\subsection{Description générale}

Il s'agit d'un modèle diphasique à phases séparées par des interfaces dans lequel les écoulements sont calculés dans les deux milieux eau et air par un modèle NavierStokes, avec un modèle de turbulence de type $k-\varepsilon$, et le suivi des interfaces est assuré par une méthode de type VOF [2] qui est présentée ci-après.

Le calcul de l'écoulement est réalisé simultanément dans l'eau et l'air afin de prendre en compte les interactions dynamiques aux interfaces entre les deux milieux. Les équations sont résolues sur un maillage curviligne et multidomaine. Le schéma numérique, qui repose sur une technique de pseudo-compressibilité et un schéma de discrétisation en temps et en espace de type volumes finis d'ordre deux, est particulièrement robuste et permet de prendre en compte des écarts de densité importants entre les deux milieux (ici de l'ordre de 1000). Une description plus détaillée du modèle numérique pourra être trouvée dans [3].

\subsection{Modélisation du déferlement avec la méthode VOF}

\subsubsection{Principe du VOF}

La méthode VOF, particulièrement adaptée pour simuler les écoulements à interfaces fortement déformées, consiste à définir à chaque instant la position de la surface libre de l'écoulement dans un maillage fixe. On utilise pour cela une fonction discrète qui représente dans chaque cellule la fraction volumique de l'eau par rapport au volume 
total de la cellule (figure 1). Les cellules liquides et gazeuses (air) sont définies respectivement par $\mathrm{VOF}=1$ et $\mathrm{VOF}=0$, et une interface liquide-air est représentée dans une cellule mixte par une valeur du VOF comprise strictement entre 0 et 1.

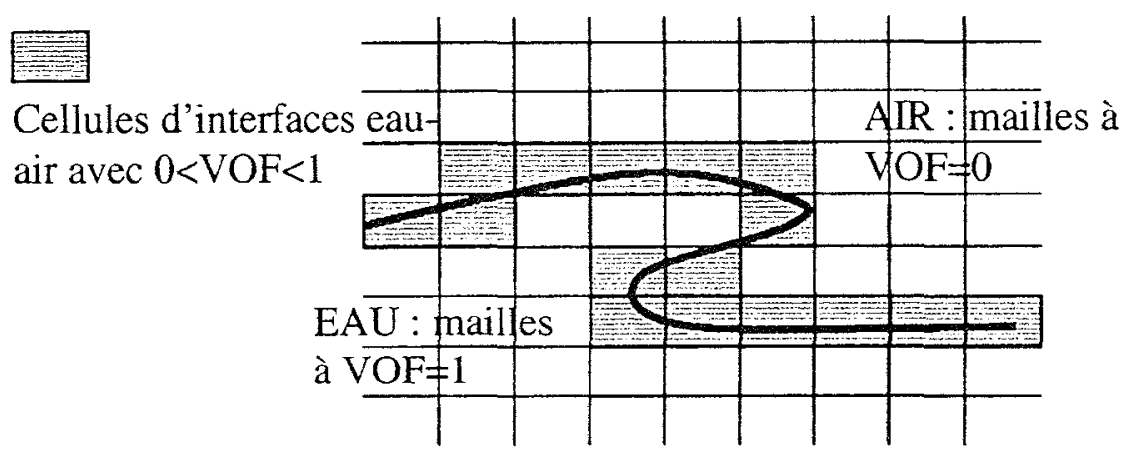

Figure 1 : Représentation schématique d'une interface dans un maillage avec un modèle VOF

Schematic representation of an interface in a mesh with a VOF model

\subsubsection{La méthode VOF standard}

Dans la méthode VOF standard [2], l'évolution de la fonction VOF est gouvernée par une équation de transport classique faisant intervenir des bilans de masse entre les cellules mixtes qui contiennent la surface libre. Le calcul des flux de masse aux interfaces (donc du bilan de masse dans chacune des cellules), qui utilise le champ de vitesse donné par intégration des équations de Navier-Stokes, est réalisé en supposant que la surface libre dans la maille ne peut prendre que deux positions (en 2D), horizontale ou verticale. Connaissant le champ de VOF dans le maillage à un instant donné, l'équation de bilan, appliquée globalement aux cellules d'interface, permet de déterminer à l'instant suivant un nouveau champ de VOF et donc une nouvelle position de la surface libre.

Cependant, la résolution de cette équation de conservation de la masse entre cellules d'interface entraîne généralement des problèmes numériques significatifs liés à l'hypothèse assez grossière sur la position verticale ou horizontale de la surface libre dans une cellule. Il en résulte alors des pertes de précision sur les valeurs de VOF données par l'équation, et donc sur la forme des interfaces, d'autant plus grandes que l'écoulement et la forme de la surface libre sont complexes, comme par exemple dans le cas du déferiement.

\subsubsection{La méthode SL-VOF (Segment Lagrangien-VOF):}

Dans le cadre d'une collaboration entre PRINCIPIA et le LSEET, une nouvelle méthode VOF a été développée [3] dans le but d'améliorer le concept de la méthode VOF de base précédemment décrite. 
La surface libre est ici représentée dans chaque cellule par un segment dont l'orientation est quelconque, contrairement à la méthode VOF standard. Au cours d'un pas de temps, le déplacement de la surface libre est réalisé en advectant de manière lagrangienne les segments dans le maillage (figure 2). Après advection, la surface libre dans chaque cellule est reconstruite par l'intermédiaire d'un segment dont la position est la moyenne des positions de tous les segments contenus dans le cellule.

La nouvelle position des segments permet alors de calculer les nouvelles valeurs du VOF après advection.

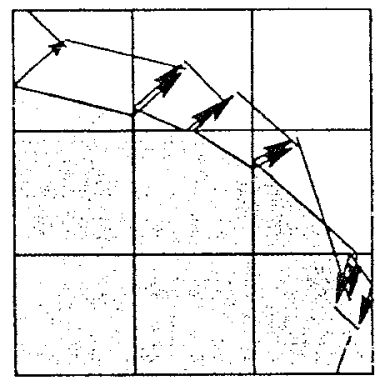

Interface initiale puis advection des segments Initial interface then advection of segments
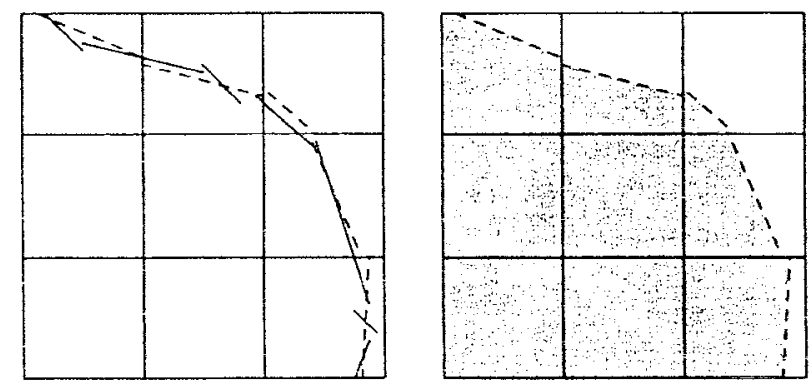

Reconstruction de l'interface après advection Interface reconstruction after advection

Figure 2 : Principe de la méthode SL-VOF $S L$-VOF method principle

Par rapport à la méthode VOF standard, la méthode SL-VOF permet des gains importants en précision et en temps de calcul ; ces derniers peuvent être diminués d'un rapport 5 environ.

\section{Résultats}

\subsection{Exemples de validation}

On présente ici quelques exemples de validation de calculs de déferlement d'une vague, d'amplitude $\mathrm{H}=0.45 \mathrm{~m}$, sur une plage de pente constante à $1 / 15$.

La vague du large est modélisée par une onde de type « onde solitaire » dont l'intérêt est relatif à la simplicité des conditions limites à imposer, avec des vitesses nulles loin de la crête. Les conditions initiales (figure 3) concernent les profils de vitesse, pression et forme de la surface libre de l'onde [4].

Les résultats obtenus avec EOLE sont comparés d'une part avec un modèle de type potentiel [1], reconnu pour sa grande précision lorsque la surface libre de la vague n'est pas trop déformée, et d'autre part avec des mesures obtenues dans le canal à houle de l'Institut des Sciences de l'Ingénieur de Toulon et du Var [3].

On note une très bonne concordance des résultats sur les formes que prend la vague déferlante lors des différentes phases du processus de déferlement : 
- au point de déferlement lorsque la crête de la vague présente une tangente verticale (figure 4), où les résultats du modèle sont comparés à une simulation obtenue avec un modèle potentiel,

- au cours de la phase de déferlement (figure 5), avec des comparaisons à des mesures en laboratoire,

- lors des phénomènes de splash-up et de swash (figure 6).

Figure 3:

Conditions initiales [4]

Initial conditions

Figure 4:

Interface au point de déferlement Interface shape at breaking point

Figure 5 :

Interface au cours du déferlement Interface shape during breaking
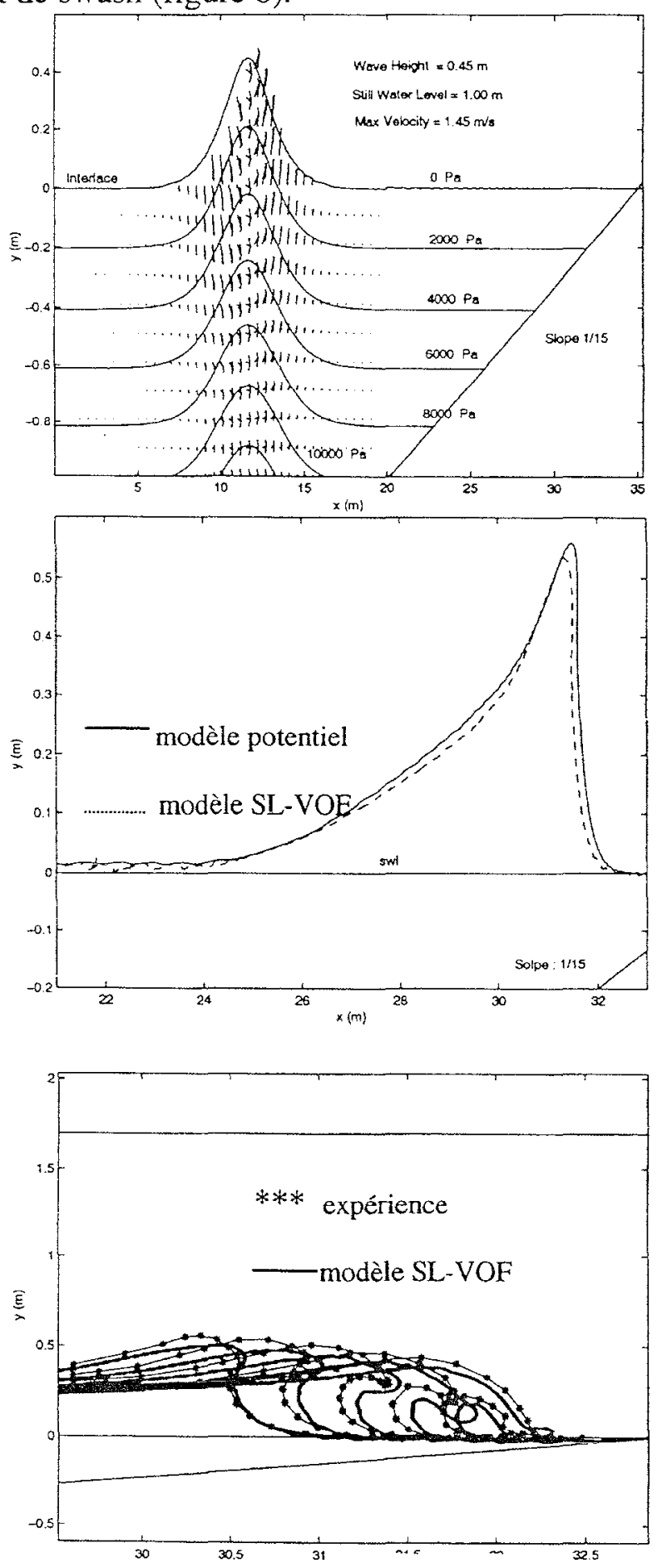
Figure 6 : Phénomènes de splash-up et swash Splash-up and swash phenomena
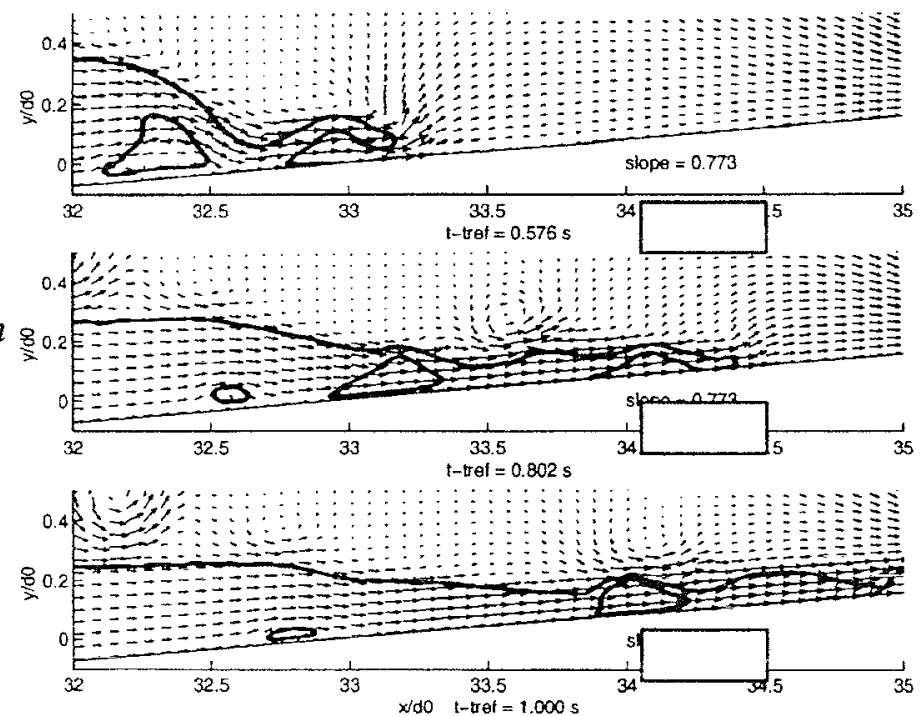

\subsection{Déferlement sur des structures côtières}

Des calculs de déferlement d'une onde solitaire d'amplitude $\mathrm{H}=8 \mathrm{~m}$ sur des structures côtières émergées sont présentés. Le domaine de calcul est composé de deux sousdomaines : le premier de $200^{*} 100$ mailles pour la propagation de la vague en amont de l'obstacle, et le second de $40 * 40$ mailles pour le suivi de la vague après son déferlement sur la structure. Trois cas, caractérisés par différentes hauteurs de francbord de la structure $h=1 \mathrm{~m}, \mathrm{~h}=2,5 \mathrm{~m}$ et $\mathrm{h}=6 \mathrm{~m}$, sont étudiés (figure 7 ).

L'amplitude de la vague atteint un maximum dans la zone d'impact sur la structure. L'influence d'une augmentation de la hauteur de la structure $h$ se traduit par une augmentation de l'amplitude maximale absolue (Hmax), mais s'accompagne d'une diminution de l'amplitude relative $(\mathrm{Hrel}=\mathrm{Hmax}-h)$ de la vague par rapport à la structure. Des différences significatives sur la forme de la vague sont également notées (figure 8). La figure 9 montre un exemple de calcul de propagation de la vague après son déferlement sur la structure, ainsi que l'impact du front d'eau sur un obstacle placé à $55 \mathrm{~m}$ plus en aval.

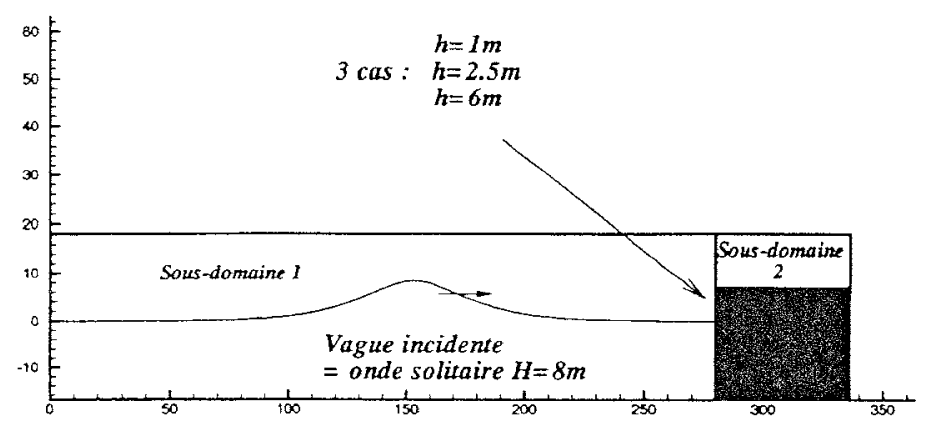

Figure 7 : Etude du déferlement d'une vague sur une structure côtière Study of wave-breaking on a coastal structure 

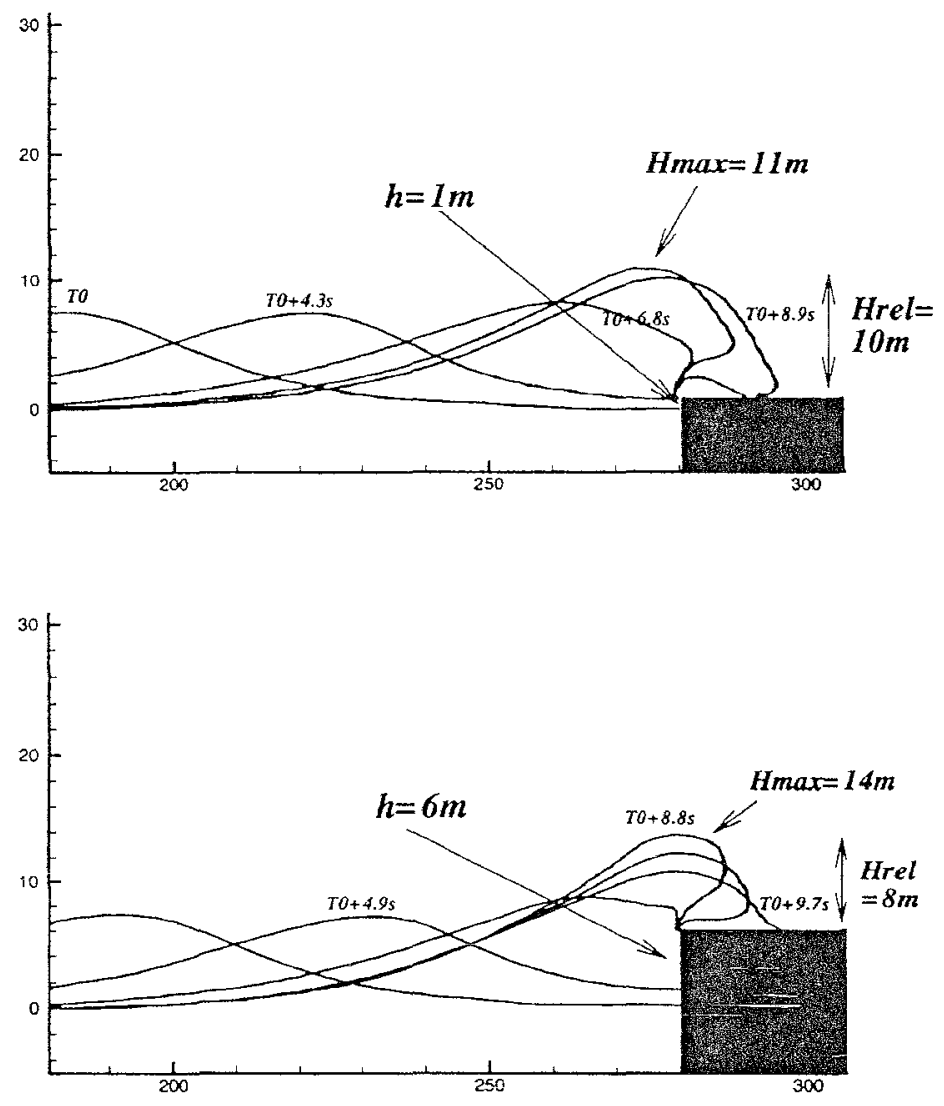

Figure 8: Déferlement pour différentes hauteurs de la structure $(\mathrm{h}=1 \mathrm{~m}$ et $6 \mathrm{~m})$ Wave-breaking for different height of the structure $(h=1 \mathrm{~m}$ and $6 \mathrm{~m})$

\section{Conclusions}

Un modèle numérique de type Navier-Stoke diphasique, avec une méthode VoF pour le suivi d'interfaces fortement non-lineaires, a été développé.

Des calculs de déferlement de vagues sur des plages (validés sur des mesures) et de franchissement et d'impact sur des structures côtières, montrent les larges potentialités et domaine de validité de ce modèle qui est capable de simuler des écoulements complexes, caractérisés par des grandes déformations des interfaces eau-air.

Le domaine d'application du modèle est large et peut couvrir différents secteurs de l'ingénierie côtière et offshore : franchissement de digues, efforts hydrodynamiques sur les structures fixes ou flottantes, problèmes d'érosion littorale induits par le déferlement de vagues,...

L'extension 3D du modèle SL-VOF est en phase finale de développement et de validation. Le principal problème de l'utilisation de ce modèle $3 \mathrm{D}$ est lié à l'augmentation importante des temps de calcul. Pour contourner cette difficulté, un couplage du modèle Navier-Stokes-VOF avec un modèle potentiel [1] est en cours de 
réalisation. Il permettra à terme d'étudier des cas réels 3D de déferlement de vagues périodiques ou aléatoires.

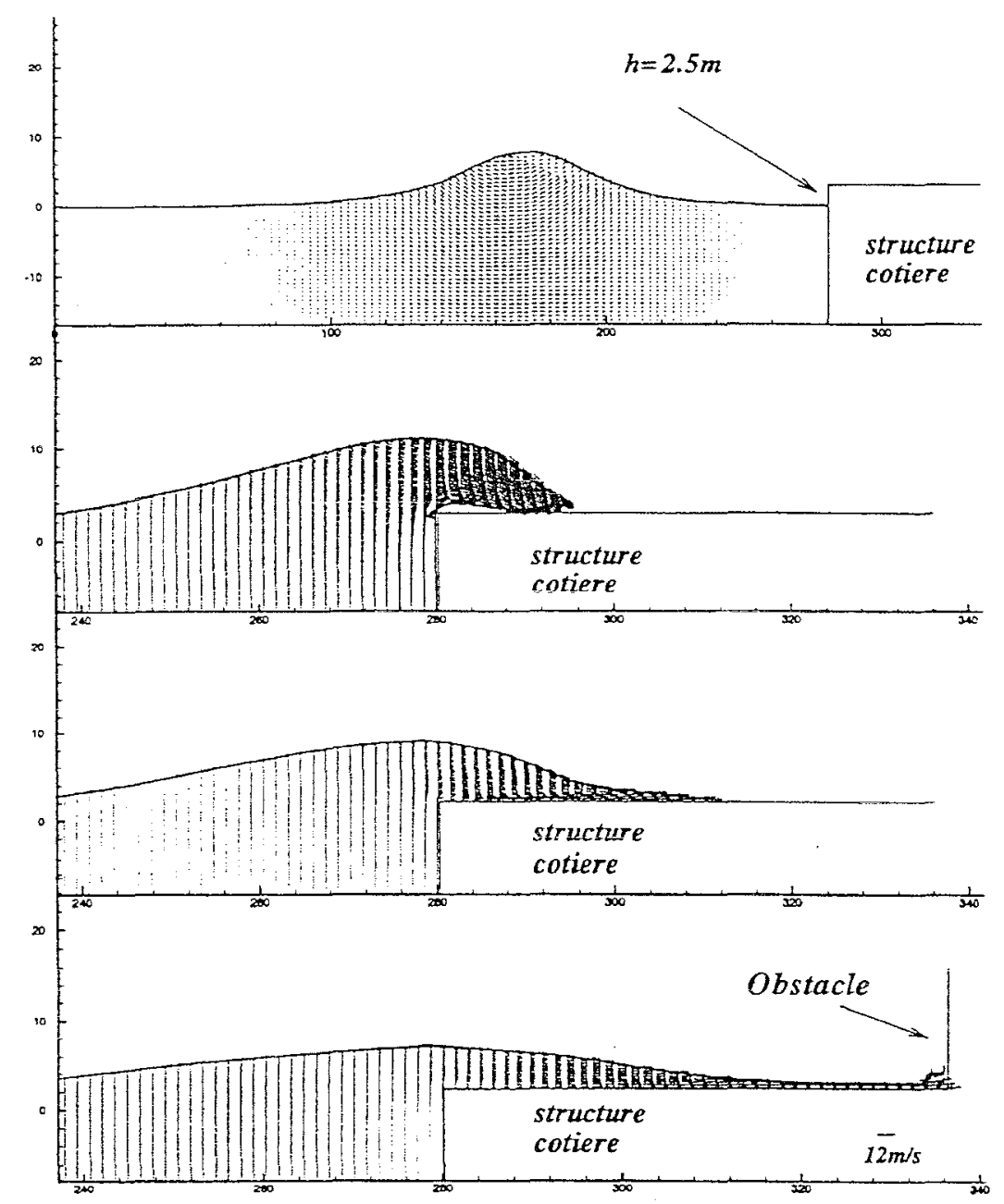

Figure $9:$ Déferlement et impact de la vague sur un obstacle en aval Wave-breaking and impact on an dowstream obstacle

\section{Références}

[1] GRILLI S.T., SVENDSEN I.A. et SUBRAMANYA (1997) - Breaking criterion and characteristics for solitary wave breaking on slopes, J. of Waterway, Port, Coastal and Ocean Eng., May-June, pp 102-112.

[2] HIRT C.W. et NICHOLS B.D. (1981) - Volume Of Fluid (VOF) Method for the Dynamics of Free Boundaries, J. of Comp. Phys., Vol. 39, pp. 201-225.

[3] GUIGNARD S., MARCER R., REY V., FRAUNIE P. (1999) - Solitary wave breaking on sloping beaches: two phase flow numerical simulation by SL-VOF method, accepted to Eur. J. of Mec.

[4] TANAKA M. (1986) - The stability of solitary waves, Phys. of Fluids 29 (3), pp 650-655. 